\title{
Development and quality assurance of computer programs for the review of long-term safety analysis
}

\author{
Jens Eckel ${ }^{1, \mathrm{a}}$, Martin Navarro ${ }^{1, \mathrm{a}}$, and Stephan Hotzel ${ }^{1, \mathrm{a}}$ \\ ${ }^{1}$ Federal Office for the Safety of Nuclear Waste Management (BASE), Wegelystrasse 8, 10623 Berlin, Germany \\ ${ }^{\text {a }}$ formerly at: GRS gGmbH, Schwertnergasse 1, 50667 Cologne, Germany
}

Correspondence: Jens Eckel (jens.eckel@bfe.bund.de)

Published: 10 November 2021

\begin{abstract}
During the site selection process, regulated by the Site Selection Act (Standortauswahlgesetz StandAG), the implementer has to identify adequate siting regions and has to perform long-term safety analysis for these regions. The Federal Office for the Safety of Nuclear Waste Management (Bundesamt für die Sicherheit der nuklearen Entsorgung - BASE) as the responsible federal authority has to review the implementer's longterm safety analysis. To perform this duty in the required depth, it will be necessary to recalculate important aspects of the analysis by means of numerical computer programs. In addition, this will allow assessment of the underlying uncertainties of the implementer's long-term safety analysis from a regulatory point of view.

Numerical modelling requires a high degree of quality assurance. Therefore, it is of vital importance that the same problem is modelled with different computer programs and - if possible - by different teams of modelers. This strategy is known as the diverse modelling approach. The goal of this approach is to perform a cross-check of the computer programs in use as well as of the correctness of data handling, data interpretation, and model implementation. Comparing outcomes of different modelling teams also integrates complementary views and approaches, which can be beneficial when dealing with complicated problems. The diverse modelling approach forms the basis of regulatory modelling. It can also be implemented within organizations if more than one code is used to tackle the same task.

The diverse modelling approach has been carried out at GRS gGmbH over the past two decades by means of the computer programs, TOUGH2-GRS and MARNIE(2). Both (co-)developed programs are thermohydraulic codes that compute transport phenomena in porous media and can be coupled to geochemical codes, see Navarro (2018) and Navarro et al. (2021) for further details. TOUGH2-GRS and MARNIE(2) were applied in various projects, such as preliminary safety analysis Gorleben (VSG, Vorläufige Sicherheitsanalyse Gorleben), ZIESEL and EVEREST. A recent example for the diverse modelling approach was the use of TOUGH2-GRS and MARNIE for the development of indicators for the safe confinement of radionuclides in a deep geological repository (Navarro et al., 2019).

A high degree of quality assurance is also achieved by involving computer programs in benchmarks to compare results among different codes for well-defined problems. For the calculation of THM (thermo-hydraulicalmechanical) processes TOUGH2-GRS has been coupled with the geomechanical computer program FLAC3D and this approach has been used in the benchmark BenVaSim (Seher et al., 2019).

In this contribution selected works related to TOUGH2-GRS and MARNIE(2) are presented. From this starting-point, ideas and concepts for the continuation of development and quality assurance of the two computer programs at BASE in the near future are outlined. These works will contribute to strengthen the capabilities of BASE in the independent review process of implementer's long-term safety analysis within the site selection process.
\end{abstract}

Kurzfassung. Während des Standortauswahlverfahrens, geregelt im Standortauswahlgesetz (StandAG), muss der Antragsteller entsprechende Standortregionen ermitteln und Langzeitsicherheitsanalysen für diese Regionen 
durchführen. Das Bundesamt für die Sicherheit der nuklearen Entsorgung (BASE) als die verantwortliche Regulierungsbehörde muss die Langzeitsicherheitsanalysen des Antragstellers überprüfen. Um diese Aufgabe in der erforderlichen Tiefe zu erfüllen, ist es notwendig, wichtige Aspekte der Analysen mithilfe numerischer Rechenprogramme nachzuvollziehen. Darüber hinaus ermöglicht dieses Vorgehen, die zugrunde liegenden Ungewissheiten der Langzeitsicherheitsanalysen des Antragstellers aus regulatorischer Perspektive zu beurteilen.

Numerische Modellierung erfordert ein hohes Maß an Qualitätssicherung. Daher ist es von essentieller Bedeutung, dass das gleiche Problem mit verschiedenen Rechenprogrammen modelliert wird und - wenn möglich - von verschiedenen Modellierteams. Diese Strategie wird als diversitärer Modellierungsansatz bezeichnet. Das Ziel dieses Ansatzes besteht darin, eine Gegenprüfung der verwendeten Computerprogramme sowie der Korrektheit der Datenverarbeitung, der Dateninterpretation und der Modellumsetzung durchzuführen. Durch den Vergleich der Ergebnisse verschiedener Modellierteams werden komplementäre Ansichten und Ansätze integriert, was von Vorteil sein kann, wenn es um komplexe Problemstellungen geht. Der diversitäre Modellierungsansatz bildet die Grundlage für die regulatorische Modellierung und kann auch innerhalb von Organisationen eingesetzt werden, wenn mehr als ein Rechenprogramm verwendet wird, um die gleiche Aufgabe zu lösen.

Der diversitäre Modellierungsansatz ist in den vergangenen 20 Jahren bei der GRS gGmbH mithilfe der Computerprogramme TOUGH2-GRS und MARNIE(2) verfolgt worden. Beide (weiter-)entwickelten Programme sind thermohydraulische Rechenprogramme, die Transportphänomene in porösen Medien berechnen und mit geochemischen Rechenprogrammen gekoppelt werden können, siehe Navarro (2018) und Navarro et al. (2021) für weitere Details. TOUGH2-GRS und MARNIE(2) wurden in verschiedenen Projekten angewendet, beispielsweise in der VSG (Vorläufige Sicherheitsanalyse Gorleben), ZIESEL und EVEREST. Ein aktuelles Beispiel für den diversitären Modellierungsansatz war die Anwendung von TOUGH2-GRS und MARNIE für die Entwicklung von Indikatoren für den sicheren Einschluss von Radionukliden in einem tiefen geologischen Endlager (Navarro et al., 2019). Ein hohes Maß an Qualitätssicherung wird auch durch Einbeziehung von Computerprogrammen in Benchmark-Projekten erzielt, um die Ergebnisse zwischen verschiedenen Rechenprogrammen für genau eingegrenzte Problemstellungen zu vergleichen. Für die Berechnung von thermohydromechanischen (THM-)Prozessen wurde TOUGH2-GRS mit dem geomechanischen Computerprogramm FLAC3D gekoppelt, und dieser Ansatz wurde in dem Benchmark BenVaSim genutzt (Seher et al., 2019).

In diesem Beitrag werden ausgewählte Arbeiten mit Bezug zu TOUGH2-GRS und MARNIE(2) präsentiert. Von diesen Ausgangspunkten aus werden Ideen und Konzepte für die Fortsetzung der Entwicklung und Qualitätssicherung der beiden Computerprogramme am BASE in der nahen Zukunft skizziert. Diese Arbeiten werden dazu beitragen, die Fähigkeiten des BASE im unabhängigen Überprüfungsprozess der Langzeitsicherheitsanalysen des Antragsstellers innerhalb des Standortauswahlverfahrens zu stärken.

\section{References}

Navarro, M.: User Manual, TOUGH2-GRS Version 2, TOUGH2MP-GRS Version 0, GRS-505, Gesellschaft für Anlagen- und Reaktorsicherheit (GRS) gGmbH, Köln, September 2018.

Navarro, M., Weyand, T., Eckel, J., and Fischer, H.: Indikatoren zur Bewertung des Einschlusses und der Isolation mit exemplarischer Anwendung auf ein generisches Endlagersystem mit dem Wirtsgestein Tongestein, GRS-A-3985, Gesellschaft für Anlagen- und Reaktorsicherheit (GRS) gGmbH, Köln, November 2019 .
Navarro, M., Beuth, T., Bracke, G., Eckel, J., Frieling, G., Hotzel, S., Kock, I., Seher, H., and Weyand, T.: Weiterentwicklung und Qualitätssicherung von Modellierungswerkzeugen zur Durchführung und Bewertung von Sicherheitsanalysen im Standortauswahlverfahren, GRS-622, Gesellschaft für Anlagen- und Reaktorsicherheit (GRS) gGmbH, Köln, Februar 2021.

Seher, H., Beuth, T., Bracke, G., Eckel, J., Frieling, G., Hotzel, S., Kock, I., Mayer, K.-M., Navarro, M., and Weyand, T.: Szenarien für die Langzeitphase von Endlagersystemen sowie lntegritätsnachweis für das Ton- und Kristallingestein, GRS-526, Gesellschaft für Anlagen- und Reaktorsicherheit (GRS) gGmbH, Köln, August 2019. 